\title{
Frequency and Timing Offset Analysis in OFDM using GNU Radio
}

\author{
G. Yamuna \\ M.Tech student \\ ECE Department \\ CVR College of Engineering
}

\author{
T. Padmavathi \\ Senior Assistant professor \\ ECE Department \\ CVR College of Engineering
}

\author{
Varghese Thattil \\ Prof \& Head of ECE \\ Department \\ CVR College of Engineering
}

\begin{abstract}
To enhance the performance and reduce the Inter Symbol Interference (ISI) at enhanced data rates in wireless communications, Orthogonal Frequency Division Multiplexing (OFDM) is being used. The performance of OFDM is degraded due to frequency and timing offsets which increase the Bit Error Rate (BER) in the wireless communication. Frequency offsets are caused due to difference between transmitter / receiver oscillators and Doppler shift. Timing offsets are due to symbol timing and sampling clock drift. This is estimated and compensated in the receiver.

In this paper packet based data transmission test bed has been implemented for OFDM to analyze frequency and time deviation. The effect of frequency offset and timing offset in packet reception over different modulation techniques like BPSK, QPSK is evaluated. By incorporating the noise voltage, normalized frequency and sample timing offset the channel model is simulated in the noisy environment and effect of these parameters considered to maximize the throughput.
\end{abstract}

The OFDM packet based data transmission with offset correction is simulated by using GNU Radio which is free \& open-source software tool that provides signal processing blocks with a facility of modifying codes in these blocks.

\section{Keywords}

OFDM, BER, Frequency offset, Timing offset, GNU Radio, SDR.

\section{INTRODUCTION}

The scope of wireless communication is increased to a great extent because of the use of multicarrier modulation techniques, among which Orthogonal Frequency Division Multiplexing (OFDM) is one of the most popular techniques.

OFDM is a modulation technique that is being used for many of the latest wireless and telecommunications standards. It allows many users to transmit in an allocated band, by subdividing the available bandwidth into many narrow bandwidth carriers [1]. Each user is allocated several carriers to transmit their data. The transmission is in such a way that the carriers used are orthogonal to one another [2], thus allowing them to be packed together much closer than standard frequency division multiplexing (FDM). This leads to OFDM providing a high spectral efficiency. Additionally the advantage of OFDM is, it reduces fading effect and increases the data rate in transmission [3].

When the signal exists beyond Guard Interval (GI) it leads to
Inter Symbol Interference (ISI). Doppler shift in received frequencies destroys the subcarrier's orthogonality and produces Inter Carrier Interference (ICI) [5] in the system. These ISI and ICI increases the packet losses due to which the performance of an OFDM system to be degraded.

The basic model of OFDM communication system with channel model for the transmission of data symbols to the receiver shown in Fig 1.

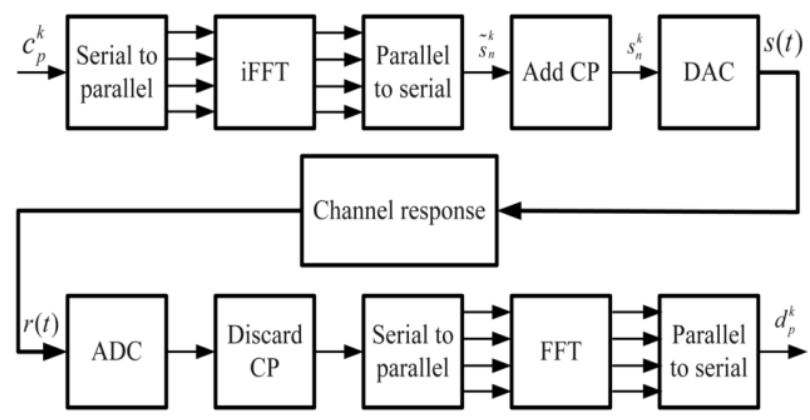

Fig 1: Block diagram of conventional OFDM system

This channel model produces LOS (Line of sight) communication and also various reflections due to which multipath effects come to the picture. To minimize the multipath effects and noise introduced by the channel, channel estimation is used. Generally, the received signal obtained through a multipath fading channel contains the channel impulse response and additive white Gaussian noise which is given by,

$$
\mathrm{R}(\mathrm{n})=\mathrm{H}(\mathrm{n}) \mathrm{C}(\mathrm{n})+\mathrm{W}(\mathrm{n}) \quad 0 \leq \mathrm{n} \leq \mathrm{N}-1
$$

Where $\mathrm{C}(\mathrm{n})$ is transmitted complex data symbol which is modulated by a BPSK or QAM modulation, $\mathrm{H}(\mathrm{n})$ is channel coefficient in frequency domain and $\mathrm{W}(\mathrm{n})$ is additive white Gaussian noise.

The channel coefficient $H(n)$ is

$$
H(n)=\sum_{j=0}^{r-1} h_{j} e^{j 2 \pi T f_{D j} \frac{n}{N} \delta\left(\lambda-\tau_{j}\right)}
$$

In equation (1.2), $\lambda$ is the delay spread index, $h_{j}$ is the complex impulse response of the $\mathrm{j}^{\text {th }}$ path, $\mathrm{f}_{\mathrm{Dj}}$ is the $\mathrm{j}^{\text {th }}$ path's Doppler frequency shift, $\tau_{j}$ is the $\mathrm{j}^{\text {th }}$ path delay time normalized by the sampling time, and $r$ is the total number of the propagation path. After removing the guard interval from $\mathrm{R}(\mathrm{n})$ the received samples are obtained and are demodulated to extract the pilot signals. The transmitted data samples $\mathrm{C}(\mathrm{n})$ 
can be recovered by simply dividing the received signal by the channel response,

$$
\mathrm{Y}(\mathrm{n})=\frac{\mathrm{R}(\mathrm{n})}{\mathrm{H}(\mathrm{n})}
$$

\subsection{Effect of Offsets and Channel Estimation}

The performance degradation in OFDM is due to frequency offset and Timing offset. Frequency offset is defined as the difference between the nominal frequency and actual output frequency. It occur due to a difference in the frequencies of the local oscillators in the transmitter and receiver, gives rise to a shift in the frequency domain which is also referred to as frequency offset $[4,5]$. This offset can also be caused due to the Doppler shift in the channel. Timing offset is occurring due to different rates between the sample clocks of the transmitter and receiver. It also occurs due to changes in symbol timing. The demodulation of a signal with these offsets can cause large BER and may degrade the performance of a symbol synchronizer. It is therefore important to estimate the frequency offset and minimize its impact. These offsets can be estimated and corrected by using channel estimator followed by equalizer.

Channel estimation is the process of analyzing / characterizing the effect of the physical medium on the transmitted data.

The channel estimation techniques are of two types, Blind channel estimation techniques and Data-aided channel estimation techniques. The first technique is used to estimate the channel state information (CSI) without the knowledge of the transmitted signal and the second estimation technique is to estimate the channel response by the known information added to the transmitted signals.

\subsection{GNU Radio Companion}

GNU Radio is a free software development toolkit $[6,7]$.It provides signal processing blocks to implement software defined radios (SDR) $[8,9]$ and signal processing systems. It can be used with external RF hardware to create SDRs or without hardware in a simulation environment. It is widely used in academic and commercial environments to support both wireless communications research and real-world radio systems [7].

Reconfigurability is a key feature with all software-defined radio systems [9]. Instead of using different radios designed for specific but different purposes, a single general-purpose radio can be used as the radio front-end, and the signalprocessing software (here GNU Radio) handles the processing specific to the radio application.

\section{OFDM IMPLEMENTATION}

The implementation of OFDM communication system include transmitter, receiver and channel model. The functionality of the OFDM communication system is verified with suitable SDR blocks shown in Fig 2.

In the transmitter the data or message signal is generated with the help of Galois linear feedback shift register (GLFSR) source. From GLFSR source Pseudo random sequence (PRC) data is encapsulated and is modified in to packet by including preamble and header. This packet is transmitted with the 64 orthogonal subcarriers. These are modulated using a modulation schemes like BPSK, QPSK etc. In this test bed BPSK modulation is used to transmit header because of its low Bit Error Rate (BER) and QPSK is used for payload. Among 64 subcarriers, 48 subcarriers are used for data, 4 subcarriers are used to carry pilot symbols. To avoid ISI and

delay spread $25 \%$ of total subcarriers are allocated for cyclic prefix $(\mathrm{CP})$. Along with $\mathrm{CP}$, preamble is used for detection and synchronization and initial channel state information (CSI).

After modulation the entire parallel data converted into serial form and then transmitted through the AWGN channel model. In the testbed normalized frequency and sample timing offsets are incorporated.

In this testbed data aided channel estimation technique is used. It can be implemented in couple of ways namely block type and comb type. The block type channel estimation is done through training symbols (sending known information over one or more whole OFDM symbols without any data), and in comb type pilots aided channel estimation (sending known information together with the data). Block type suits more for slow-fading channels and comb type is used for fast fading channels.

At the receiver side preamble is used to detect the frame starting time and offset which occur due to multipath by using Schmidl \& Cox technique [10]. It uses two training symbols with special pattern. With the help of first symbol it produce a trigger signal to find the frame starting time. The time domain preamble proposed by Schmidl \& Cox technique is

$$
\operatorname{Preamble}(\mathrm{p})=\left[\begin{array}{ll}
\mathrm{P}_{\frac{\mathrm{N}}{2}} & \mathrm{P}_{\frac{\mathrm{N}}{2}}
\end{array}\right]
$$

Where, $\mathrm{P}_{\mathrm{N} / 2}$ represents samples of length $\mathrm{N} / 2$. The timing metric of Schmidl \& Cox is given as

$$
M(d)=\frac{|P(d)|^{2}}{(R(d))^{2}}
$$

Where,

$$
\begin{gathered}
\mathrm{P}(\mathrm{d})=\sum_{k=0}^{\frac{N}{2}-1} r^{*}(d+k) \cdot r\left(d+k+\frac{N}{2}\right) \\
\mathrm{R}(\mathrm{d})=\sum_{k=0}^{\frac{N}{2}-1}\left|r\left(d+k+\frac{N}{2}\right)\right|^{2}
\end{gathered}
$$

The starting point of symbol is given by the maximum of timing metric $M(d)$. The timing metric of Schmidl \& Cox has a large plateau and has its peak for entire interval of CP. Frequency offset can also be calculated and corrected with the Schmidl \& Cox technique [10]. The trigger signal along with received data is send to header and payload Demultiplexer (HPD) block. The HPD block copies the header and preamble information to its first output. The header data is demodulated to identify the packet number and payload length and feedback to the HPD block. Based on this information HPD copies the payload to its second output and it also demodulated. From the preambles and pilot sequences the channel estimation calculates the frequency offset with the Schmidl \& Cox technique. The calculated frequency Offsets is corrected in the serial equalizer block.

Serializer plucks the data symbols from the occupied carriers and outputs them as a stream of complex scalars. Constellation decoder decodes constellation's points from a complex space to (unpacked) bits based on the map of the constellation object. Payload demodulation follows the same procedure as header demodulation except channel estimation block 


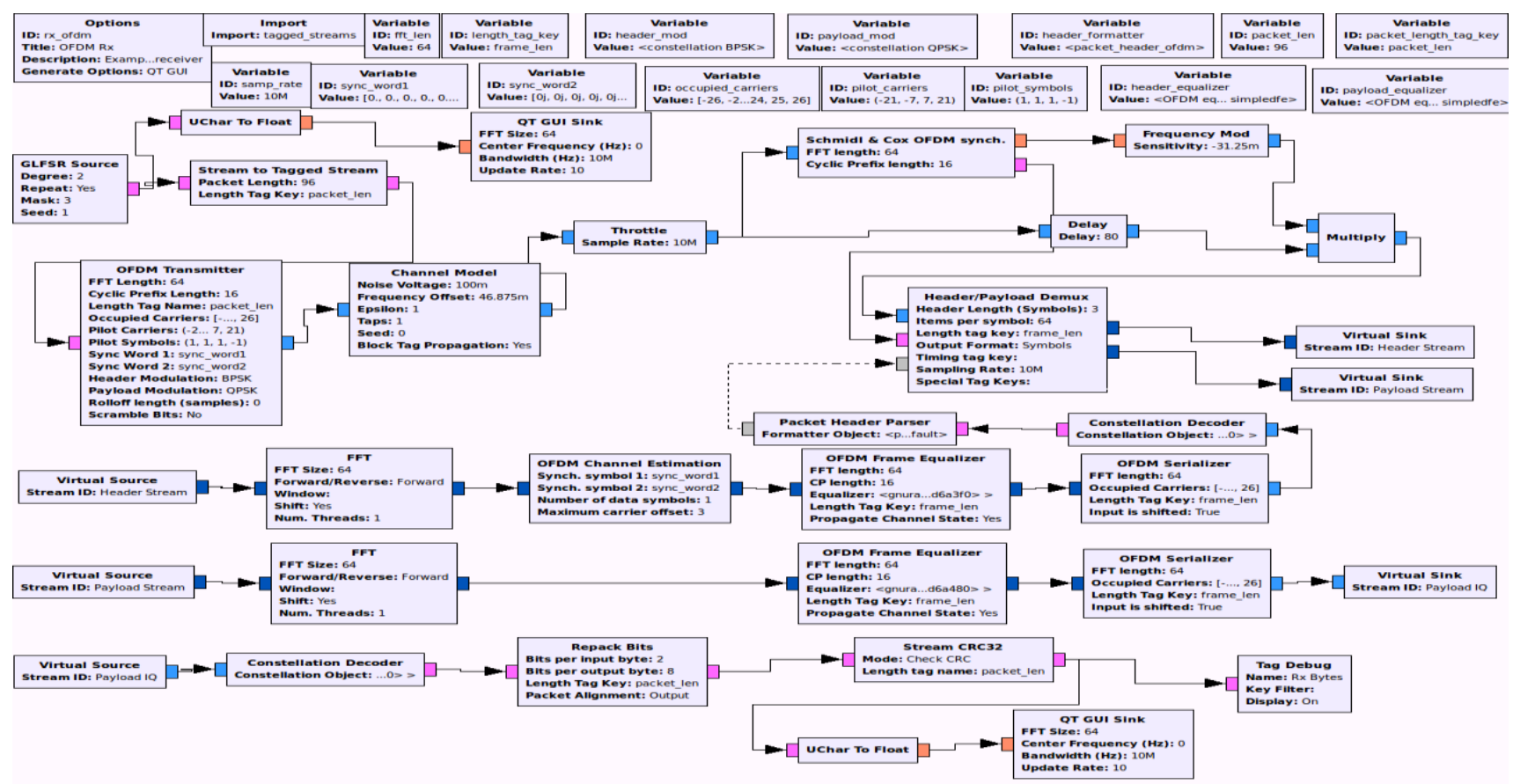

Fig 2: Block diagram of OFDM system

\section{EXPERIMENTAL RESULTS}

The required parameters and specifications for OFDM transmitter are defined in Table 1. The IFFT/FFT size is 64 and cyclic prefix is $1 / 4$ th of the IFFT/FFT size (FFT size $=64$ and CP length=16 (1/4th of 64)). The cyclic prefix reduces the problem caused due to delay spread and maintains continuity of the signal which ensures orthogonal reception of received signal subcarriers.

Table 1: Experimental Parameters

\begin{tabular}{|c|c|}
\hline Parameters & Specifications \\
\hline FFT Size & 64 \\
\hline Occupied tones & 52 \\
\hline Cyclic prefix & 16 \\
\hline Sampling Rate & $10 \mathrm{M}$ \\
\hline Modulation technique & BPSK, QPSK \\
\hline Sub carrier spacing & $156.25 \mathrm{KHz}$ \\
\hline $\begin{array}{c}\text { System Channel } \\
\text { Bandwidth }\end{array}$ & $8 \mathrm{MHz}$ \\
\hline
\end{tabular}

The GLFSR source generates the input data with polynomial degree of 2 , with peak to peak amplitude of +1 to -1 which is shown in Fig 3.

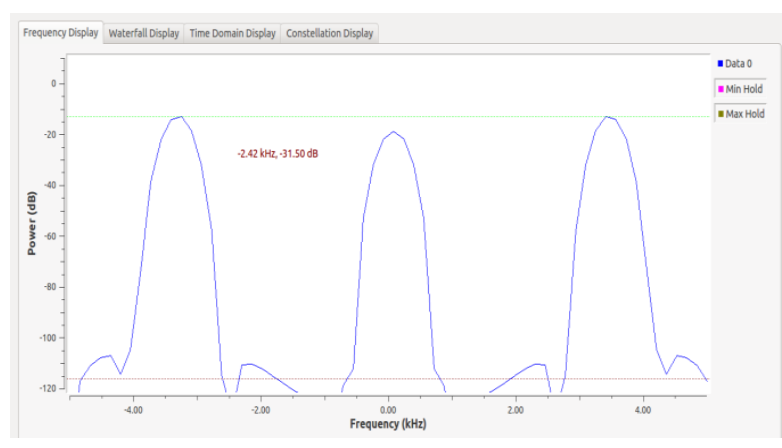

Fig 3: Frequency domain format of input signal

In this testbed 64 orthogonal carriers are used to modulate the data. The resultant time domain representation of OFDM signal is shown in Fig 4.

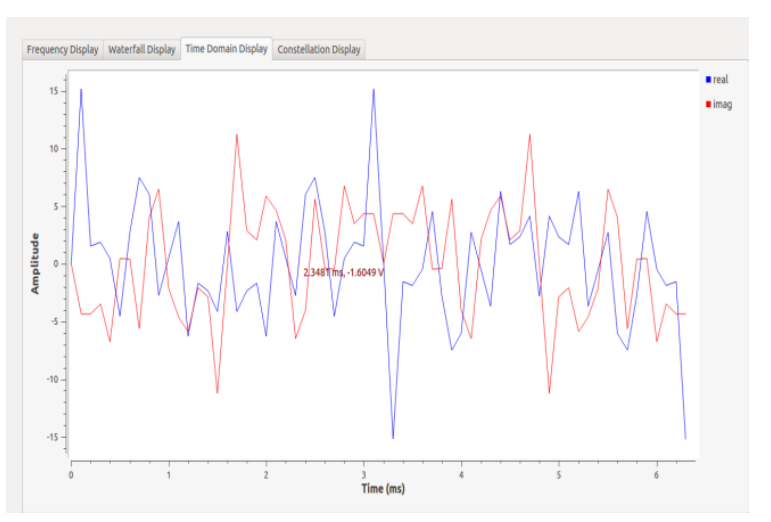

Fig 4: Time domain representation of OFDM signal

The functionality of OFDM Transmission and reception is verified by observing the demodulated signal at the output of receiver.The frequency domain representation of both signals are displayed in Fig 5. 


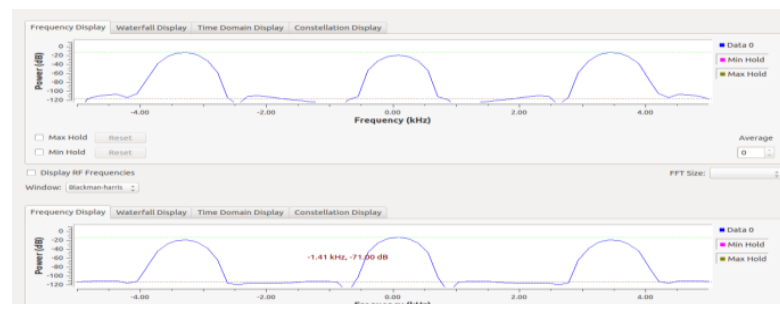

Fig 5: Frequency domain representation of transmitted and received signals

\subsection{Effect of Frequency Offset in Packet Reception}

The channel model used in this testbed allows the user to incorporate the noise voltage, a normalized frequency offset and a sample timing offset of AWGN source. By using these parameters the OFDM testbed is simulated in noisy environment. The effect of these parameters in packet reception can be shown in table 2 by varying these offsets slowly.

An increase of offset in the channel model causes loss of packets. Due to this frequency offset resulting throughput is less even for 50 packets without any channel estimation. With the help of frequency offset parameter in channel model the offset is inserted in the carrier slowly and the effect of this offset is observed in the packet reception as shown in table 2 . Sub carrier spacing used in this test bed is $156.25 \mathrm{KHz}$.

Table 2: Effect of Frequency Offset in Packet Reception

\begin{tabular}{|c|c|}
\hline Frequency Offset (Hz) & $\begin{array}{c}\text { Number of Packets lost } \\
\text { during Transmission }\end{array}$ \\
\hline 0 (no offset) & Nil \\
\hline 3.90625 & Nil \\
\hline 11.7188 & Nil \\
\hline 14.0625 & Loss in 28 packets \\
\hline 15.625 & Loss in 35 packets \\
\hline 15.6406 & Loss in 45 packets \\
\hline 15.6562 & All \\
\hline 15.6719 &
\end{tabular}

The effect of frequency offset in packet reception is shown in Fig 6.

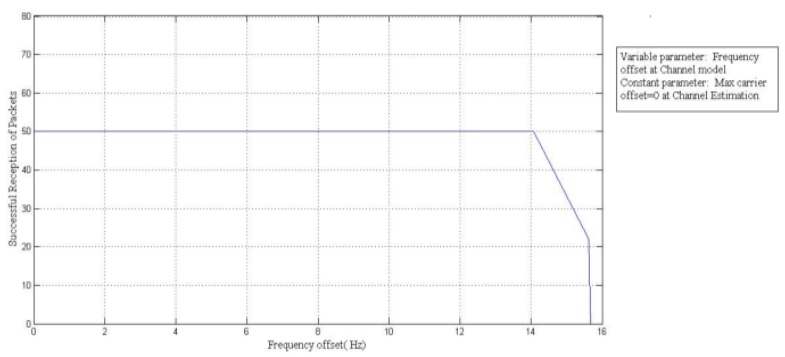

Fig 6: Effect of Frequency Offset in Packet Reception
The above figure 6 indicates maximum number of packets can be received at lower offset levels. Packets received successfully until the frequency offset level of $14.0625 \mathrm{~Hz}$. If the offset is higher than $14.0625 \mathrm{~Hz}$ packet losses occurred.

However this loss can be controlled to certain extent with the help of Channel Estimator block in this testbed and throughput is maximized.

\subsection{Performance of Channel Estimation}

By including OFDM Channel Estimation in the OFDM system the coarse frequency offset correction has been done with the help of Equalizer. The max_carrier_offset parameter set at 3 (means offset 3 is shared by 64 carriers, each carrier get $46.8 \mathrm{~Hz}$ offset). If the offset occur in channel for each carrier is smaller than $46.8 \mathrm{~Hz}$, packets may receive successfully. If it is greater than $46.8 \mathrm{~Hz}$ packet loss may occur. It is observed by keeping max_carrier_offset at 3 i.e. constant and the frequency offset parameter in channel model is varied. It can also be verified with max_carrier_offset parameter set at 5 (means offset 5 is shared by 64 carriers, each carrier get $78.12 \mathrm{~Hz}$ offset). If the offset occur in channel for each carrier is smaller than $78.12 \mathrm{~Hz}$, packets may receive successfully. If it is greater than $78.12 \mathrm{~Hz}$ packet loss may occur. It is observed by keeping max_carrier_offset at 5 i.e. constant and the frequency offset parameter in channel model is varied.

The effect of Frequency Offset in Packet Reception with Channel Estimation is shown in table 3.

Table 3: The effect of Frequency Offset in Packet Reception with Channel Estimation

\begin{tabular}{|c|c|c|c|}
\hline \multicolumn{2}{|c|}{ Max_carrier_offset at 3 } & \multicolumn{2}{c|}{ Max_carrier_offset at 5 } \\
\hline $\begin{array}{c}\text { Frequency } \\
\text { Offset (Hz) }\end{array}$ & $\begin{array}{c}\text { Number of } \\
\text { Packets lost } \\
\text { during } \\
\text { Transmission }\end{array}$ & $\begin{array}{c}\text { Frequency } \\
\text { Offset(Hz) }\end{array}$ & $\begin{array}{c}\text { Number of } \\
\text { Packets lost } \\
\text { during } \\
\text { Transmission }\end{array}$ \\
\hline 0 & Nil & 0 & Nil \\
\hline 7.8125 & Nil & 7.8125 & Nil \\
\hline 15.625 & Nil & 15.625 & Nil \\
\hline 23.4375 & Nil & 31.25 & Nil \\
\hline 31.25 & Nil & 46.875 & Nil \\
\hline 39.0625 & Nil & 54.6875 & Nil \\
\hline 46.875 & $\begin{array}{c}\text { Loss in } 22 \\
\text { packets }\end{array}$ & 62.5 & Nil \\
\hline 46.8906 & $\begin{array}{c}\text { Loss in } 37 \\
\text { packets }\end{array}$ & 78.125 & $\begin{array}{c}25 \text { packets } \\
\text { are lost }\end{array}$ \\
\hline 46.9062 & $\begin{array}{c}\text { Loss in } 47 \\
\text { packets }\end{array}$ & 78.1406 & $\begin{array}{c}39 \text { packets } \\
\text { are lost }\end{array}$ \\
\hline 46.9219 & All & 78.1562 & $\begin{array}{c}48 \text { packets } \\
\text { are lost }\end{array}$ \\
\hline
\end{tabular}

The effect of frequency offset in packet reception is shown in Fig 7 and 8. 


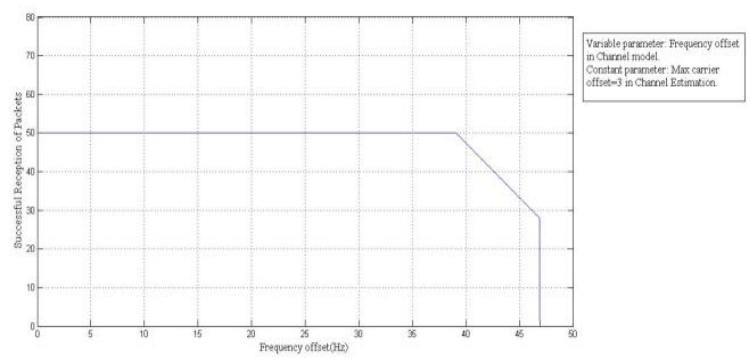

Fig 7: Effect of Frequency Offset in Packet Reception with Channel Estimation.

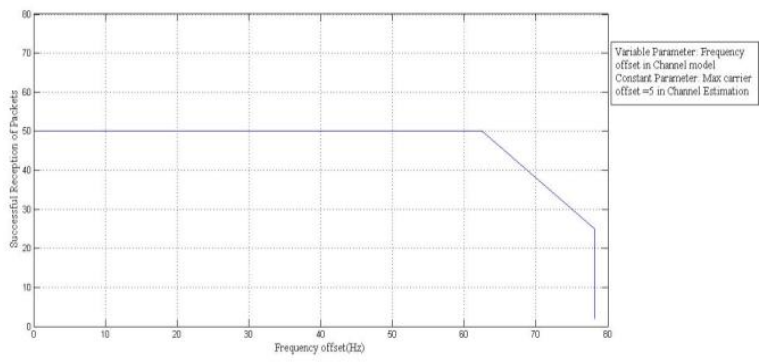

Fig 8: Effect of Frequency Offset in Packet Reception with Channel Estimation.

From the Fig 6, 7 and 8, it is verified that the packets can be received successfully even at higher offsets with the help of Channel Estimation. Packets are received successfully until the carriers getting offset of $39.0625 \mathrm{~Hz}$ when the max_carrier offset at each carrier is $46.8 \mathrm{~Hz}$. If the max_carrier offset at each carrier is $78.12 \mathrm{~Hz}$ packets received successfully until each carrier has an offset of $62.5 \mathrm{~Hz}$

Packet loss in OFDM system with and without channel estimation is observed and correspondingly plots are drawn. The Fig 9 shows that the packet loss with channel estimation is lesser when compared to one without channel estimation.

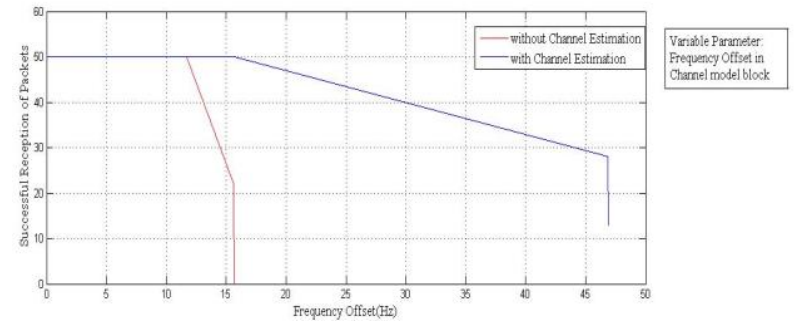

Fig 9: Comparison between with $\&$ without channel estimation in terms of successful packet reception.

\subsection{Effect of Jitter (Timing Offset) in Packet Reception}

Along with the frequency offset the effect of timing offset in packet reception has verified as shown in table 4. It indicates that an exponential rise in offset leads the loss of packet. In this testbed with the help of epsilon parameter in channel model increase the timing offset and verified this effect on packet reception is shown in table 4 .
Table 4: Effect of Timing Offset in Packet Reception

\begin{tabular}{|c|c|}
\hline Timing Offset & $\begin{array}{c}\text { Number of Packets lost } \\
\text { during Transmission }\end{array}$ \\
\hline 1 & Nil \\
\hline 1.001 & Nil \\
\hline 1.002 & Nil \\
\hline 1.0022 & 6 packets loss \\
\hline 1.0023 & 18 packets loss \\
\hline 1.0024 & 27 packets loss \\
\hline 1.0025 & 41 packets loss \\
\hline
\end{tabular}

Timing offset at the level 1 indicates that there is no difference between sample clocks of the transmitter and receiver. It is observed that if the difference is above the level of 1.002 loss of packets occur. The study has been carried out at $\mathrm{CP}=16$ (25\% of FFT size). The effect of Timing offset in packet reception is shown in figure 10 .

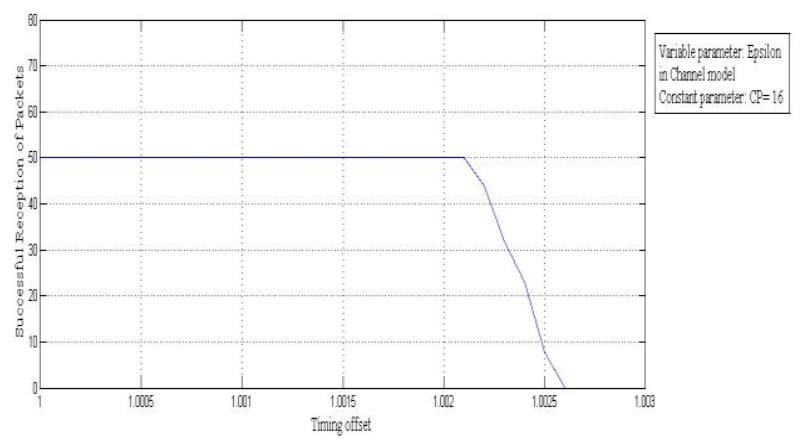

Fig 10: Effect of Timing Offset in Packet Reception

Carrier alignment used in the packet structure is total of 64 carriers among that positive carriers 1 to 26 and negative carriers -1 to -26 carrying data and remaining carriers along with dc carrier does not carry any data to reduce the out of band radiation as shown below.

Tag Debug: Rx Bytes

Input Stream: 00

Offset: 0 Source: n/a Key: ofdm_sync_chan_taps Value: $\#\left[\begin{array}{lllllll}(0,0) & (0,0) & (0,0) & (0,0) & (0,0) & (0,0) & (-61.2248,19.2573)\end{array}\right.$ $41.9073,48.8774) \quad(-7.04559,63.2687) \quad(30.0647,55.6706)$ $\begin{array}{lll}(57.7198,30.0126) & (63.1257,-11.4095) \quad(49.1463,-40.9515)\end{array}$ $\begin{array}{llll}(17.8219,-60.9554) & (-19.0174,-59.8843) & (-49.9789,-40.6941)\end{array}$ $\begin{array}{llll}(-64.417,-5.63722) & (-57.8558,30.182) & (-29.5168,57.0675)\end{array}$ $\begin{array}{lll}(7.09093,64.4349) & (41.0565,49.4044) \quad(61.8325,17.9921)\end{array}$ $(60.1552,-18.4434)(4.23578,-5.28534)(0.64715,-6.47385)(-$ $34.4013,-53.0797)(-6.88902,-2.56118)(-7.41967,1.53169)(-$ $5.46137,5.32159) \quad(-2.5624,6.67435) \quad(2.2246,6.81144)$ $(5.1245,3.9951)(0,0)(6.14543,-3.82864)(3.53023,-6.4351)(-$ $1.04827,-7.01485)(-4.24902,-5.65356)(-6.66226,-2.49364)(-$ $6.56885,2.05846) \quad(-36.2451,53.0564) \quad(-0.77507,7.85118)$ $\begin{array}{llll}(3.14919,5.95864) & (6.06372,3.97592) & (6.41652,-0.757187)\end{array}$ $(5.79803,-5.13304)(1.29741,-7.46136)(-2.58211,-6.87754)(-$ $6.26773,-4.02898)(-6.99886,-0.856864)(-6.49312,3.2603)(-$ $2.75139,6.45069) \quad(0.97933,6.91148) \quad(4.04979,6.74923)$ $(61.113,13.532) \quad(6.70458,-2.20793) \quad(5.16285,-4.94544)$ $\begin{array}{lll}(1.81976,-7.58057) & (-3.44643,-6.41928) & (-6.17859,-4.1949)\end{array}$ $(0,0)(0,0)(0,0)(0,0)(0,0)]$

Offset: 0 Source: n/a Key: packet_num Value: 0 
Offset: 0 Source: n/a Key: ofdm_sync_carr_offset Value: 0

Offset: 0 Source: n/a Key: packet_len Value: 96

\section{CONCLUSION}

In this paper packet based, OFDM testbed is implemented using GNU platform and the performance of OFDM system is evaluated in the simulation environment. All the blocks of OFDM have been thoroughly investigated by giving different parameters as inputs.

The effect of frequency and timing offsets in packet reception is observed by varying these offset levels in channel model. The simulation results show that increase in offset causes increases in BER which leads loss of packets. The frequency offset error is detected and corrected with the help of Schmidl \& Cox technique, channel estimation and equalization techniques respectively and throughput is maximized. These simulation results show that OFDM channel estimation using Schmidl \& Cox Technique is effective till the stage of hardware/ spectrum limitations.

The development of the OFDM testbed with SDR opens various opportunities to research further advanced signal processing techniques for OFDM based systems, such as the improved synchronization and channel estimation techniques, the techniques to reduce the well known Peak-to-Average Power Ratio (PAPR) issue, and the emerging Multiple-Input Multiple-Output (MIMO) technique. To make use of the OFDM channel estimation testbed in real time using USRP, can be extended as future work to observe the bit error rate (BER). This can also verified by using different modulation schemes such as QPSK, QAM, 8 QAM, instead of BPSK and BER can also be reduced with suitable synchronization schemes.

\section{REFERENCES}

[1] Nicola Marchetti, Muhammad Imadur Rahman, Sanjay Kumar and Ramjee Prasad "OFDM: Principles and Challenges"
[2] Understanding an OFDM transmission: http://www.dsplog.com/2008/02/03/understanding- anofdm transmission/

[3] Manushree Bhardwaj, Arun Gangwar, Devendra Soni "A Review on OFDM: Concept, Scope \& its Applications" IOSR Journal of Mechanical and Civil Engineering (IOSRJMCE) ISSN: 2278-1684 Volume 1, Issue 1 (May-June 2012), PP 07-11 www.iosrjournals.org.

[4] Mourad MELLITI ${ }^{+}$, Salem HASNAOUI ${ }^{+}$, Ridha BOUALLEGUE ${ }^{++}$, "Analysis of Frequency Offsets and Phase Noise Effects on an OFDM 802.11 g Transceiver", ${ }^{(+)}$SYSCOM Laboratory, National School of Engineering of Tunis TUNISIA ${ }^{(++)}$SYSTEL Laboratory SUP'COM, National School of Engineering of Sousse TUNISIA IJCSNS VOL.7 No.4, April 2007.

[5] Inter Carrier Interference (ICI) in OFDM due to Frequency offset http://www.dsplog.com/2009/08/08/effect-of-ici-inofdm/

[6] Ke-Yu, Chen (8818-0493), Zhi-Feng Chen (12181197), "GNU Radio" Dept. of Electrical Computer Engineering University of Florida, Gainesville, Florida.

[7] WhatIsGR \& what exactly does GNU Radio do from https://gnuradio.org/redmine/projectGnuradio/wiki/What IsGR

[8] "What is Software Defined Radio -Wireless Innovation Form"from www.wirelessinnovation.org/what_is_sdr.

[9] Carlos E. Caicedo, Ph.D. Student. Software Defined Radio and Software Radio Technology: Concepts and Application. Department of Information Science and Telecommunications University of Pittsburgh.

[10] T. M. Schmidl, and D. C. Cox, "Robust frequency and timing synchronization for OFDM," IEEE Trans.Comm. 45, 1613-1621 (1997). 